PROCEEDINGS OF THE

AMERICAN MATHEMATICAL SOCIETY

Volume 129, Number 6, Pages 1775-1783

S 0002-9939(00)05974-8

Article electronically published on November 21, 2000

\title{
THE ASYMPTOTIC BEHAVIOR OF A CLASS OF NONLINEAR DELAY DIFFERENCE EQUATIONS
}

\author{
HASSAN SEDAGHAT AND WENDI WANG \\ (Communicated by Michael Handel)
}

Abstract. The asymptotic behavior of difference equations of type

$$
x_{n}=x_{n-1}^{p}\left[1+g\left(\sum_{i=1}^{m} f_{i}\left(x_{n-i}\right)\right)\right], \quad p>0,
$$

is studied, where $g$ and each $f_{i}$ are continuous real functions with $g$ decreasing and $f_{i}$ increasing. Results include sufficient conditions for permanence, oscillations and global attractivity.

\section{INTRODUCTION}

In this paper we study the asymptotic behavior of nonlinear delay difference equations of type

$$
x_{n}=x_{n-1}^{p}\left[1+g\left(\sum_{i=1}^{m} f_{i}\left(x_{n-i}\right)\right)\right], \quad p \in(0, \infty), n=1,2,3, \ldots,
$$

where it is assumed that the initial values are non-negative and the following hypotheses hold:

(H1) $\quad g: \mathbb{R} \rightarrow(-1, \infty)$ is continuous and decreasing with $g(0)=0$;

(H2) For $i=1, \ldots, m$, the functions $f_{i}:[0, \infty) \rightarrow \mathbb{R}$ are continuous and non-decreasing with the sum $f=\sum_{i=1}^{m} f_{i}$ increasing.

Equation (11) originated as an economic model. In [2], economists W. Baumol and E. Wolff presented a model for analyzing the productivity growth of the research and development (R\&D) sector of the economy. In [5], this model was extended to include possible time delays in the integration of $\mathrm{R} \& \mathrm{D}$ output by the heterogeneous client industry. Conditions implying permanence and bounded oscillations were presented in [5] for this version of the Baumol-Wolff model.

The results that we obtain here extend those in [5] in a number of ways; for instance, in addition to the introduction of $p$ in (1), we no longer require continuous differentiability of $g$ and the $f_{i}$. Our results include, in particular, the characterization of behavior near the origin as well as conditions implying permanence, global attractivity and other types of asymptotic behavior. As might be expected, the three cases $0<p<1, p=1$ and $p>1$ give rise to substantially different dynamical behaviors with the case $p=1$ overlapping the other two.

Received by the editors October 5, 1999.

1991 Mathematics Subject Classification. Primary 39A10.

Key words and phrases. Global attractivity, persistent oscillations, permanence. 
We shall be interested only in the non-negative solutions of (11) and assume that hypotheses (H1) and (H2) hold throughout this paper. Background concepts and related developments may be found in the standard literature (e.g., [1], 3], 4]).

\section{The CASE $p \leq 1$}

Lemma 1. Equation (11) has a unique positive fixed point if either of the following hold:

(i) $p<1$;

(ii) $p=1$ and $f(\bar{x})=0$ for some $\bar{x}>0$.

Proof. First suppose that $p<1$. Then the positive fixed points of (1) are solutions of

$$
u^{1-p}=1+g(f(u)) .
$$

The function $\varphi(u)=u^{1-p}-[1+g(f(u))]$ is continuous and increasing on $[0, \infty)$ and $\varphi(0)=-1-g(f(0))<0$. Furthermore, $\varphi(u) \geq u^{1-p}-1-g(f(0))$ so that $\varphi(u)>0$ for all sufficiently large values of $u$. Hence, (2) has a unique positive solution.

If (ii) holds, then the number $\bar{x}=f^{-1}(0)$ is clearly a solution of the equation $1=1+g(f(u))$, and thus a positive fixed point of (1). This $\bar{x}$ is unique because the function $1+g(f(u))$ is decreasing.

Lemma 2. Suppose that either condition in Lemma 1 holds. For each $n \geq 1$, if $x_{n-i} \geq \bar{x}$ (respectively, $x_{n-i} \leq \bar{x}$ ) for $i=1, \ldots, m$, then $x_{n} \leq x_{n-1}$ (respectively, $\left.x_{n} \geq x_{n-1}\right)$. The same assertions hold with all inequalities strict.

Proof. If, for some $n, x_{n-i} \geq \bar{x}$ for $i=1, \ldots, m$, then

$$
x_{n} \leq x_{n-1}^{p}[1+g(f(\bar{x}))]=x_{n-1}^{p} \bar{x}^{1-p}=\left(\frac{\bar{x}}{x_{n-1}}\right)^{1-p} x_{n-1} \leq x_{n-1} .
$$

The proof is done in the same way when inequalities are reversed or strict.

Definition 1. Equation (1) is permanent if there are positive real numbers $\alpha, \beta$ such that for every choice $x_{0}, x_{-1}, \ldots, x_{-m+1}$ of positive initial values, there is an integer $n_{0}=n_{0}\left(x_{0}, x_{-1}, \ldots, x_{-m+1}\right) \geq 1$ such that $x_{n} \in[\alpha, \beta]$ for all $n \geq n_{0}$.

Theorem 1. If either condition in Lemma 1 holds, then equation (1) is permanent.

Proof. We consider only the case $p<1$; for a proof of the other case, see [5]. For every $n \geq 1$ note that

$$
\begin{aligned}
x_{n} & \leq x_{n-1}^{p}\left[1+g\left(\sum_{j=1}^{m} f_{j}(0)\right)\right] \\
& \leq\left(x_{n-2}^{p}\left[1+g\left(\sum_{j=1}^{m} f_{j}(0)\right)\right]\right)^{p}[1+g(f(0))] \\
& \leq x_{n-2}^{p^{2}}[1+g(f(0))]^{1+p} .
\end{aligned}
$$

Continuing this pattern, by induction we obtain

$$
x_{n} \leq x_{0}^{p^{n}}[1+g(f(0))]^{1+p+\cdots+p^{n-1}}=x_{0}^{p^{n}}[1+g(f(0))]^{\left(1-p^{n}\right) /(1-p)}
$$


for all $n \geq 1$. If $x_{0}>0$, then the limit of the right-hand side of (3) is

$$
\lim _{n \rightarrow \infty} x_{0}^{p^{n}}[1+g(f(0))]^{\left(1-p^{n}\right) /(1-p)}=[1+g(f(0))]^{1 /(1-p)}
$$

regardless of the actual value of $x_{0}$. Define

$$
\beta=[1+g(f(0))]^{1 /(1-p)}
$$

and note that if $\left\{x_{n}\right\}$ is any solution of (II) that is generated by positive initial values, then there is a positive integer $n_{0}$ such that $x_{n} \in(0, \beta+1)$ for all $n \geq n_{0}$. Furthermore, since

$$
\beta>[1+g(f(\bar{x}))]^{1 /(1-p)}=\left[\bar{x}^{1-p}\right]^{1 /(1-p)}=\bar{x},
$$

defining $\gamma=[1+g(f(\beta+1))] /(\beta+1)^{1-p}$, we see that

$$
\gamma<\frac{1+g(f(\bar{x}))}{\bar{x}^{1-p}}=1
$$

Now, for all $n \geq n_{0}+m$,

$$
x_{n}=\frac{1+g\left(\sum_{i=1}^{m} f_{i}\left(x_{n-i}\right)\right)}{x_{n-1}^{1-p}} x_{n-1}>\frac{1+g(f(\beta+1))}{(\beta+1)^{1-p}} x_{n-1}=\gamma x_{n-1} .
$$

Now consider two possible cases: (I) there is a positive integer $k \geq n_{0}+m$ such that $x_{k} \geq \bar{x}$, or (II) $x_{n}<\bar{x}$ for all $n$.

In Case (I), inequality (4) implies that

$$
x_{k+1}>\gamma x_{k} \geq \gamma \bar{x} .
$$

It follows inductively that $x_{k+m}>\gamma^{m} \bar{x}$. If $n>k+m$ and $x_{n-i} \leq \bar{x}$ for $i=1, \ldots, m$, then, by Lemma $2, x_{n} \geq x_{n-1}$. Hence, $x_{n} \geq \gamma^{m} \bar{x}$ for all $n>k+m$ and permanence is established.

In Case (II), again by Lemma $2,\left\{x_{n}\right\}$ is an increasing sequence that must therefore converge to $\bar{x}$; thus for all sufficiently large $n$, it must be true that $x_{n} \in\left[\gamma^{m} \bar{x}, \beta\right]$ and permanence follows.

Definition 2. A solution $\left\{x_{n}\right\}$ of (1) oscillates persistently if $\left\{x_{n}\right\}$ is bounded and has two or more limit points.

The following corollary extends an analogous result in [5].

Corollary 1. Assume that the functions $g, f_{i}$ are continuously differentiable and that either one of the conditions in Lemma 1 holds. If the fixed point $\bar{x}$ is a repeller (i.e., all roots of the characteristic equation of (1) at $\bar{x}$ have modulus greater than 1), then every non-trivial, positive solution of (1) oscillates persistently in the interval of Theorem 1.

Proof. Given the similarity of the problem to that in [5], we need only show here that, when $p<1$, no positive solution of (11) can be constantly equal to $\bar{x}$ after a finite number of steps. To this end, assume on the contrary that there is a positive integer $k$ such that $x_{n}=\bar{x}$ for all $n \geq k$. Then, in particular,

$$
\bar{x}=x_{k+m-1}=\bar{x}^{p}\left[1+g\left(f(\bar{x})-f_{m}(\bar{x})+f_{m}\left(x_{k-1}\right)\right)\right]
$$

which, since $\bar{x}$ satisfies (2), implies that

$$
g(f(\bar{x}))=g\left(f(\bar{x})-f_{m}(\bar{x})+f_{m}\left(x_{k-1}\right)\right) .
$$

The function $g$ being monotonic, we must have $f_{m}(\bar{x})=f_{m}\left(x_{k-1}\right)$. Also, since $\bar{x}$ is a repeller, it is the case that $f_{m}^{\prime}(\bar{x})>0$, so therefore, $\bar{x}=x_{k-1}$. Applying the same 
argument inductively, it follows that $x_{n}=\bar{x}$ for all $n<k$ as well, so that $\left\{x_{n}\right\}$ had to be the trivial solution $\bar{x}$. The rest of the proof proceeds in the same manner as that in [5].

Theorem 2. Assume that either one of the conditions in Lemma 1 hold. The fixed point $\bar{x}>0$ of (11) is a global attractor of all positive solutions if for $p<1$ the equation

$$
\bar{x}^{p^{m}}\left[1+g\left(f\left(\bar{x}^{p^{m}}[1+g(f(x))]^{\left(1-p^{m}\right) /(1-p)}\right)\right)\right]^{\left(1-p^{m}\right) /(1-p)}=x,
$$

and for $p=1$ the equation

$$
\bar{x}\left[1+g\left(f\left(\bar{x}[1+g(f(x))]^{m}\right)\right)\right]^{m}=x
$$

has a unique solution in $[0, \bar{x}]$, namely, $\bar{x}$ in each case.

Proof. If $\left\{x_{n}\right\}$ is any positive solution of (1), we show that $\lim _{n \rightarrow \infty} x_{n}=\bar{x}$. There are three possible cases:

Case I. There is a positive integer $N$ such that $x_{n} \geq \bar{x}$ for all $n \geq N$; i.e., $\left\{x_{n}\right\}$ is eventually greater than or equal to $\bar{x}$. Then by Lemma $2, x_{n} \leq x_{n-1}$ for all $n \geq N+m$, i.e., $\left\{x_{n}\right\}$ is eventually non-increasing. Thus, $\bar{x}$ being the unique fixed point, it follows that $\lim _{n \rightarrow \infty} x_{n}=\bar{x}$.

Case II. $\left\{x_{n}\right\}$ is eventually less than or equal to $\bar{x}$. In this case, again Lemma 2 implies that $\left\{x_{n}\right\}$ is eventually non-decreasing and so we must once again have $\lim _{n \rightarrow \infty} x_{n}=\bar{x}$.

Case III. $\left\{x_{n}\right\}$ oscillates about $\bar{x}$. Define

$$
\lambda=\liminf _{n \rightarrow \infty} x_{n}, \quad \mu=\limsup _{n \rightarrow \infty} x_{n} .
$$

By Theorem 1,

$$
0<\lambda \leq \mu<\infty
$$

We now show that $\lambda=\bar{x}=\mu$ under the hypotheses of this theorem. By (77), for each $\varepsilon>0$ there is a positive integer $N_{\varepsilon}$ such that

$$
\lambda-\varepsilon \leq x_{n} \leq \mu+\varepsilon
$$

for all $n \geq N_{\varepsilon}$. Let

$$
\left\{x_{k+1}, x_{k+2}, \ldots, x_{k+r}\right\} \quad \text { and } \quad\left\{x_{k+r+1}, x_{k+r+2}, \ldots, x_{k+r+s}\right\}
$$

represent, respectively, a negative and a positive semicycle of $\left\{x_{n}\right\}$, where $k>$ $N_{\varepsilon}+m$. If

$$
x_{k+i_{1}}=\min _{1 \leq j \leq r}\left\{x_{k+j}\right\}
$$

with $1 \leq i_{1} \leq r$ the least integer with this property, then, by Lemma $2, i_{1} \leq m$. Similarly, if

$$
x_{k+r+i_{2}}=\max _{1 \leq j \leq s}\left\{x_{k+r+j}\right\}
$$

with $1 \leq i_{2} \leq s$ the least integer with this property, then Lemma 2 implies $i_{2} \leq m$. 
Now, assume that $p<1$ and note that, using (8), we have

$$
\begin{aligned}
x_{k+i_{1}} & =x_{k+i_{1}-1}^{p}\left[1+g\left(\sum_{j=1}^{m} f_{j}\left(x_{k+i_{1}-j}\right)\right)\right] \\
& \geq\left(x_{k+i_{1}-2}^{p}\left[1+g\left(\sum_{j=1}^{m} f_{j}\left(x_{k+i_{1}-1-j}\right)\right)\right]\right)^{p}[1+g(f(\mu+\varepsilon))] \\
& \geq x_{k+i_{1}-2}^{p^{2}}[1+g(f(\mu+\varepsilon))]^{1+p} .
\end{aligned}
$$

Continuing in this fashion and noting that $x_{k} \geq \bar{x}$, we obtain after $i_{1}$ steps

$$
x_{k+i_{1}} \geq \bar{x}^{p^{i_{1}}}[1+g(f(\mu+\varepsilon))]^{\left(1-p^{i_{1}}\right) /(1-p)} .
$$

Since $i_{1} \leq m$, it follows that $1>p \geq p^{i_{1}} \geq p^{m}$ and thus

$$
1 \leq \frac{1-p^{i_{1}}}{1-p} \leq \frac{1-p^{m}}{1-p}
$$

Re-writing the right-hand side of (9) as

$$
\bar{x}\left[\frac{1+g(f(\mu+\varepsilon))}{\bar{x}^{1-p}}\right]^{\left(1-p^{i_{1}}\right) /(1-p)}=\bar{x}\left[\frac{1+g(f(\mu+\varepsilon))}{1+g(f(\bar{x}))}\right]^{\left(1-p^{i_{1}}\right) /(1-p)}
$$

and noting that the ratio in the brackets on the right is less than 1, (9) and (10) yield

$$
x_{k+i_{1}} \geq \bar{x}\left[\frac{1+g(f(\mu+\varepsilon))}{1+g(f(\bar{x}))}\right]^{\left(1-p^{m}\right) /(1-p)}=\bar{x}^{p^{m}}[1+g(f(\mu+\varepsilon))]^{\left(1-p^{m}\right) /(1-p)} .
$$

A similar calculation shows that

$$
x_{k+r+i_{2}} \leq \bar{x}^{p^{m}}[1+g(f(\lambda-\varepsilon))]^{\left(1-p^{m}\right) /(1-p)} .
$$

So, upon letting $\varepsilon$ decrease to zero, we obtain

$$
x_{k+i_{1}} \geq \bar{x}^{p^{m}}[1+g(f(\mu))]^{\left(1-p^{m}\right) /(1-p)}
$$

and

$$
x_{k+p+i_{2}} \leq \bar{x}^{p^{m}}[1+g(f(\lambda))]^{\left(1-p^{m}\right) /(1-p)}
$$

for every sufficiently large $k$. Since the semicycles were chosen arbitrarily, we conclude that

(11) $\lambda \geq \bar{x}^{p^{m}}[1+g(f(\mu))]^{\left(1-p^{m}\right) /(1-p)}$ and $\mu \leq \bar{x}^{p^{m}}[1+g(f(\lambda))]^{\left(1-p^{m}\right) /(1-p)}$.

Next, define

$$
h(x)=\bar{x}^{p^{m}}[1+g(f(x))]^{\left(1-p^{m}\right) /(1-p)}, \quad x \in[0, \infty),
$$

and note that $h(\bar{x})=\bar{x}$. Given that $\lambda \leq \bar{x}$, if equality holds, then by (11) $\mu \leq \bar{x}$ and the proof is complete. So suppose that $\lambda<\bar{x}$. Due to the decreasing nature of $h$, we find that

$$
h^{2}(\lambda)=h(h(\lambda)) \leq h(\mu) \leq \lambda .
$$

On the other hand, $h^{2}(\bar{x})=\bar{x}$, and by (15), this is the only solution of the equation $h^{2}(x)=x$ in $[0, \bar{x}]$. Since

$$
h^{2}(0)=\bar{x}^{p^{m}}\left[1+g\left(f\left(\bar{x}^{p^{m}}[1+g(f(0))]^{\left(1-p^{m}\right) /(1-p)}\right)\right)\right]^{\left(1-p^{m}\right) /(1-p)}>0,
$$


we conclude that $h^{2}(x)>x$ for all $x<\bar{x}$. In particular, $h^{2}(\lambda)>\lambda$, which contradicts (12) and completes the proof for the case $p<1$. The proof for $p=1$ is analogous if we notice that $p^{m}=1$ and $\left(1-p^{m}\right) /(1-p)=1+p+\cdots+p^{m-1}=m$.

Corollary 2 below gives a more intuitive interpretation of the conditions in Theorem 2 ; it says that when the functions $f_{i}, g$ have sufficiently small slopes, then $\bar{x}$ is globally asymptotically stable. We first state a lemma on local stability which is proved similarly to an analogous result in [5].

Lemma 3. Assume that the functions $g, f_{i}$ are continuously differentiable. Under the conditions of Lemma 1, the fixed point $\bar{x}$ is locally asymptotically stable if

$$
c f^{\prime}(\bar{x})<1-p+2 \min \left\{p, c f_{1}^{\prime}(\bar{x})\right\} \text { where } c=\bar{x}^{p}\left|g^{\prime}(f(\bar{x}))\right| .
$$

In the next corollary, $\left(1-p^{k}\right) /(1-p)=k$ when $p=1$.

Corollary 2. Assume that the functions $g, f_{i}$ are continuously differentiable. Under the conditions of Lemma $1, \bar{x}$ is a stable global attractor of positive solutions if the following conditions are satisfied:

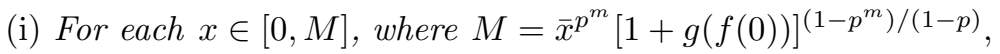

$$
\left|g^{\prime}(f(x)) f^{\prime}(x)\right|<\frac{(1-p)[1+g(f(0))]}{M\left(1-p^{m}\right)} ;
$$

(ii) $c f^{\prime}(\bar{x})<1-p+2 c f_{1}^{\prime}(\bar{x})$, where $c$ is defined in (13).

Proof. Define

$$
\alpha=\frac{M\left(1-p^{m}\right)}{(1-p)[1+g(f(0))]} \sup _{0 \leq x \leq M}\left|g^{\prime}(f(x))\right| f^{\prime}(x)
$$

so that $\alpha<1$ by (14) and the compactness of $[0, M]$. Let $h$ be the function defined in the proof of Theorem 2. Then

$$
\left|h^{\prime}(x)\right| \leq \frac{1-p^{m}}{1-p} \bar{x}^{p^{m}}[1+g(f(0))]^{\left(p-p^{m}\right) /(1-p)}\left|g^{\prime}(f(x))\right| f^{\prime}(x) \leq \alpha
$$

for all $x \in[0, M]$. Hence,

$$
|h(x)-\bar{x}|=|h(x)-h(\bar{x})| \leq \alpha|x-\bar{x}|
$$

for all $x \in[0, M]$. From this, it follows that if $x^{*} \in[0, \bar{x}]$ and $h^{2}\left(x^{*}\right)=x^{*}$, then

$$
\left|x^{*}-\bar{x}\right|=\left|h\left(h\left(x^{*}\right)\right)-\bar{x}\right| \leq \alpha^{2}\left|x^{*}-\bar{x}\right|
$$

which is possible only when $x^{*}=\bar{x}$. Hence, Theorem 2 implies that $\bar{x}$ is globally attracting. It remains to show stability. Using the fact that

$$
\bar{x}^{p^{m}}=\bar{x}^{p}\left(\frac{1}{\bar{x}^{1-p}}\right)^{\left(p-p^{m}\right) /(1-p)}=\bar{x}^{p}\left(\frac{1}{1+g(f(\bar{x}))}\right)^{\left(p-p^{m}\right) /(1-p)},
$$

the inequality in (i) for $x=\bar{x}$ yields

$$
\bar{x}^{p}\left|g^{\prime}(f(\bar{x}))\right| f^{\prime}(\bar{x})<\frac{1-p}{\left(1-p^{m}\right)}\left[\frac{1+g(f(\bar{x}))}{1+g(f(0))}\right]^{\left(p-p^{m}\right) /(1-p)}<1 .
$$

Now, (15) and (ii) imply (13); hence we have local stability. 
Remark 1. In particular, Condition (i) in Corollary 2 holds if each of the derivatives $g, f_{i}^{\prime}$ is uniformly small in magnitude, i.e., if there are constants $B, A_{i}, i=1, \ldots, m$, such that $\left|g^{\prime}(x)\right| \leq B$ for all $x \in \mathbb{R},\left|f_{i}^{\prime}(x)\right| \leq A_{i}$ for all $x \in[0, M]$, and

$$
B \sum_{i=1}^{m} A_{i}<\frac{(1-p)[1+g(f(0))]}{M\left(1-p^{m}\right)} \text {. }
$$

It should be kept in mind, though, that $M$ depends on $\bar{x}$.

\section{THe CASE $p \geq 1$}

Through linearization, it is easy to see that the origin is locally asymptotically stable if either $p>1$ or $p=1$ and $f(0)>0$. The next result provides more information without assuming differentiability.

Theorem 3. (a) If $p>1$, then the origin is asymptotically stable and attracts every trajectory of (11) with initial values in the interval $[0, \beta)$ where $\beta$ is the quantity defined in the proof of Theorem 1, namely,

$$
\beta=[1+g(f(0))]^{-1 /(p-1)} .
$$

(b) If $p=1$ and $f(0) \geq 0$, then every positive solution of (11) monotonically decreases to zero.

Proof. (a) Note that

$$
\begin{aligned}
x_{n} & =x_{n-1}^{p}\left[1+g\left(\sum_{i=1}^{m} f_{i}\left(x_{n-i}\right)\right)\right] \\
& \leq x_{n-1}^{p}[1+g(f(0))] \\
& \leq\left(x_{n-2}^{p}[1+g(f(0))]\right)^{p}[1+g(f(0))] \\
& =x_{n-2}^{p^{2}}[1+g(f(0))]^{1+p} .
\end{aligned}
$$

This pattern continues; by induction

$$
x_{n} \leq x_{0}^{p^{n}}[1+g(f(0))]^{\left(p^{n}-1\right) /(p-1)}=\beta\left(\frac{x_{0}}{\beta}\right)^{p^{n}} .
$$

Therefore, if the inital values (in particular, $\left.x_{0}\right)$ are in $[0, \beta)$, then the solution they generate must converge to zero. Stability is an immediate consequence of the monotonically decreasing nature of the sequence $\left\{\left(x_{0} / \beta\right)^{p^{n}}\right\}$.

(b) If $p=1$ and $f(0) \geq 0$, then for all $u>0$ we have $f(u)>0$ and hence $1+g(f(u))<1$. Thus, if $\left\{x_{n}\right\}$ is a positive solution of (1) , then

$$
x_{n}=x_{n-1}\left[1+g\left(\sum_{i=1}^{m} f_{i}\left(x_{n-i}\right)\right)\right]<x_{n-1}
$$

for every $n \geq 1$. Thus $\left\{x_{n}\right\}$ is monotonically decreasing and must, therefore, converge to the (unique) fixed point zero.

The next theorem may be compared with Theorems 1-3.

Theorem 4. Let $S$ be the set of all positive fixed points of (11) and let $\phi$ be the function

$$
\phi(u)=u^{p-1}[1+g(f(u))], \quad u \geq 0 .
$$


(a) If $p>1$ and $S$ is non-empty, then $\bar{x}=\inf S>0$ is an unstable fixed point; in fact, in every neighborhood of $\bar{x}$ there are initial values generating solutions that converge monotonically to zero.

(b) If $p>1$, and if $S$ is non-empty and bounded with $\phi(u) \geq 1$ for all $u>\sup S$, then $\bar{x}=\sup S$ is an unstable fixed point of (1) and in every neighborhood of $\bar{x}$ there are initial values generating unbounded, monotonically increasing solutions.

(c) If $p=1$ and $f(u)<0$ for all $u>0$, then every positive solution of (1) is unbounded and monotonically increasing.

Proof. (a) Since $S$ is the set of all solutions of $\phi(u)=1$ and $\phi$ is continuous with $\phi(0)=0$, it follows that $\bar{x}=\inf S$ is indeed a positive fixed point of (1). Now let $x_{0} \in(0, \bar{x})$ and $x_{1-m}, \ldots, x_{-1} \geq x_{0}$. Then

$$
x_{1}=x_{0}^{p}\left[1+g\left(\sum_{i=1}^{m} f_{i}\left(x_{1-i}\right)\right)\right] \leq x_{0}^{p}\left[1+g\left(f\left(x_{0}\right)\right)\right]=x_{0} \phi\left(x_{0}\right)<x_{0} .
$$

Therefore, $x_{1}<x_{0}<\bar{x}$ and $x_{2-m}, \ldots, x_{-1}, x_{0} \geq x_{1}$. It follows by induction that the sequence $\left\{x_{n}\right\}$ is monotonically decreasing in the interval $(0, \bar{x})$ so that it must converge to 0 . Since $x_{1-m}, \ldots, x_{-1}, x_{0}$ may be chosen arbitrarily close to $\bar{x}$, this also proves that $\bar{x}$ is unstable.

(b) Choose $x_{0}>\bar{x}$ and $x_{1-m}, \ldots, x_{-1}<x_{0}$ and notice that

$$
x_{1}=x_{0}^{p}\left[1+g\left(\sum_{i=1}^{m} f_{i}\left(x_{1-i}\right)\right)\right]>x_{0}^{p}\left[1+g\left(f\left(x_{0}\right)\right)\right]=x_{0} \phi\left(x_{0}\right) \geq x_{0}
$$

so that $x_{1}>x_{0}>x_{2-m}, \ldots, x_{-1}, \bar{x}$. By induction, $\left\{x_{n}\right\}$ must be monotonically increasing with $x_{n}>\sup S$ for all $n \geq 1$. Therefore, $\left\{x_{n}\right\}$ is unbounded. Since $x_{1-m}, \ldots, x_{-1}, x_{0}$ may be chosen arbitrarily close to $\bar{x}$, this also proves that $\bar{x}$ is unstable.

(c) If $f(u)<0$ for all $u>0$, then

$$
\phi(u)=1+g(f(u))>1
$$

for all $u>0$. Therefore, there are no positive fixed points and $x_{n}>x_{n-1}$ for all $n \geq 1$ if $x_{0}>0$. It follows that every positive solution must be monotonically increasing and, hence, unbounded.

The following result summarizes the stability characteristics of the origin as determined by the values of $p$.

Corollary 3. If $p>1$ or if $p=1$ and $f(0) \geq 0$, then the origin is asymptotically stable. If $p<1$ or if $p=1$ and $f(0)<0$, then the origin is unstable.

\section{REFERENCES}

[1] R.P. Agarwal, "Difference Equations and Inequalities," Dekker, New York, 1992. MR 92m:39002

[2] W.J. Baumol and E.N. Wolff, Feedback between R\&D and productivity growth: A chaos model, in: J. Benhabib (ed.) "Cycles and Chaos in Economic Equilibrium," Princeton University Press, Princeton, 1992.

[3] V.L. Kocic and G. Ladas, "Global Behavior of Nonlinear Difference Equations of Higher Order with Applications," Kluwer Academic, Boston, 1993. MR 94k:39005 
[4] J.P. LaSalle, "The Stability and Control of Discrete Processes," Springer, New York, 1986. MR 87m:93001

[5] H. Sedaghat, Effects of temporal heterogeniety in the Baumol-Wolff productivity growth model, Economic Theory, 15(2), 2000.

Department of Mathematical Sciences, Virginia Commonwealth University, RichMOND, VIRGINIA 23284-2014

E-mail address: hsedagha@vcu.edu

Department of Mathematics, Southwest Normal University, Chong Qing 400715, PeoPLE'S REPUBLIC OF CHINA

E-mail address: wendi@swnu.edu.cn 